Pacific

Journal of

Mathematics

\title{
$q$-HYPERGEOMETRIC DOUBLE SUMS AS MOCK THETA FUNCTIONS
}

\author{
JEREMY LOVEJOY AND ROBERT OSBURN
}

Volume 264 No. 1

July 2013 


\title{
$q$-HYPERGEOMETRIC DOUBLE SUMS AS MOCK THETA FUNCTIONS
}

\author{
JEREMY LOVEJOY AND ROBERT OSBURN \\ In memory of Basil Gordon
}

\begin{abstract}
Recently, Bringmann and Kane established two new Bailey pairs and used them to relate certain $q$-hypergeometric series to real quadratic fields. We show how these pairs give rise to new mock theta functions in the form of $q$-hypergeometric double sums. We also prove an identity between one of these sums and two classical mock theta functions introduced by Gordon and McIntosh.
\end{abstract}

\section{Introduction}

A Bailey pair relative to $a$ is a pair of sequences $\left(\alpha_{n}, \beta_{n}\right)_{n \geq 0}$ satisfying

$$
\beta_{n}=\sum_{k=0}^{n} \frac{\alpha_{k}}{(q)_{n-k}(a q)_{n+k}} .
$$

Here we have used the standard $q$-hypergeometric notation,

$$
(a)_{n}=(a ; q)_{n}=\prod_{k=1}^{n}\left(1-a q^{k-1}\right),
$$

valid for $n \in \mathbb{N} \cup\{\infty\}$. The Bailey lemma states that if $\left(\alpha_{n}, \beta_{n}\right)$ is a Bailey pair relative to $a$, then so is $\left(\alpha_{n}^{\prime}, \beta_{n}^{\prime}\right)$, where

$$
\alpha_{n}^{\prime}=\frac{(b)_{n}(c)_{n}(a q / b c)^{n}}{(a q / b)_{n}(a q / c)_{n}} \alpha_{n}, \quad \beta_{n}^{\prime}=\sum_{k=0}^{n} \frac{(b)_{k}(c)_{k}(a q / b c)_{n-k}(a q / b c)^{k}}{(a q / b)_{n}(a q / c)_{n}(q)_{n-k}} \beta_{k}
$$

Inserting (1-3) into (1-1) with $n \rightarrow \infty$ gives

$$
\sum_{n \geq 0}(b)_{n}(c)_{n}(a q / b c)^{n} \beta_{n}=\frac{(a q / b)_{\infty}(a q / c)_{\infty}}{(a q)_{\infty}(a q / b c)_{\infty}} \sum_{n \geq 0} \frac{(b)_{n}(c)_{n}(a q / b c)^{n}}{(a q / b)_{n}(a q / c)_{n}} \alpha_{n},
$$

MSC2010: primary 33D15; secondary 05A30, 11F03, $11 \mathrm{~F} 37$.

Keywords: $q$-hypergeometric series, mock theta functions, Appell-Lerch series, Hecke-type double sums. 
valid whenever both sides converge. For more on Bailey pairs, including historical perspectives and recent advances, see [Andrews 1986b, Chapter 3; 2001], or [Warnaar 2001].

In a recent study of multiplicative $q$-series, Bringmann and Kane [2011] established two new and interesting Bailey pairs. They showed that $\left(a_{n}, b_{n}\right)$ is a Bailey pair relative to 1 , where

$$
\begin{aligned}
a_{2 n} & =\left(1-q^{4 n}\right) q^{2 n^{2}-2 n} \sum_{j=-n}^{n-1} q^{-2 j^{2}-2 j}, \\
a_{2 n+1} & =-\left(1-q^{4 n+2}\right) q^{2 n^{2}} \sum_{j=-n}^{n} q^{-2 j^{2}}, \\
b_{n} & =\frac{(-1)^{n}\left(q ; q^{2}\right)_{n-1}}{(q)_{2 n-1}} \chi(n \neq 0),
\end{aligned}
$$

and $\left(\alpha_{n}, \beta_{n}\right)$ is a Bailey pair relative to $q$, where

$$
\begin{aligned}
\alpha_{2 n} & =\frac{1}{1-q}\left(q^{2 n^{2}+2 n} \sum_{j=-n}^{n-1} q^{-2 j^{2}-2 j}+q^{2 n^{2}} \sum_{j=-n}^{n} q^{-2 j^{2}}\right), \\
\alpha_{2 n+1} & =-\frac{1}{1-q}\left(q^{2 n^{2}+4 n+2} \sum_{j=-n}^{n} q^{-2 j^{2}}+q^{2 n^{2}+2 n} \sum_{j=-n-1}^{n} q^{-2 j^{2}-2 j}\right), \\
\beta_{n} & =\frac{(-1)^{n}\left(q ; q^{2}\right)_{n}}{(q)_{2 n+1}} .
\end{aligned}
$$

These closely resemble Bailey pairs related to seventh order mock theta functions [Andrews 1986a], but surprisingly no $q$-series obtained by a direct substitution of either (1-5)-(1-7) or (1-8)-(1-10) in (1-4) is a genuine mock theta function. For example, it turns out that substituting (1-5)-(1-7) in (1-4) with $b, c \rightarrow \infty$ yields

$$
\frac{-q}{(-q)_{\infty}} \omega(q)
$$

where $\omega(q)$ is one of the third order mock theta functions. The presence of the infinite product means that this is not a mock theta function but a mixed mock modular form.

Recall that mock theta functions are $q$-series which were introduced by Ramanujan in his last letter to G. H. Hardy on January 12, 1920. Until 2002, it was not known how these functions fit into the theory of modular forms. Thanks to work of Zwegers [2002] and Bringmann and Ono [2006; 2010], we now know that each of Ramanujan's examples of mock theta functions is the holomorphic part of a weight $\frac{1}{2}$ harmonic weak Maass form $f(\tau)$ (as usual, $q:=e^{2 \pi i \tau}$ where $\tau=x+i y \in \mathbb{U}$ ). Following [Zagier 2009], the holomorphic part of any weight $k$ harmonic weak 
Maass form $f$ is called a mock modular form of weight $k$. If $k=\frac{1}{2}$ and the image of $f$ under the operator $\xi_{k}:=2 i y^{k} \bar{\partial} / \partial \bar{\tau}$ is a unary theta function, then the holomorphic part of $f$ is called a mock theta function. Specializations of the Appell-Lerch series

$$
m(x, q, z):=\frac{1}{j(z, q)} \sum_{r \in \mathbb{Z}} \frac{(-1)^{r} q^{\left(\begin{array}{c}
r \\
2
\end{array}\right)} z^{r}}{1-q^{r-1} x z}
$$

are perhaps the most well-known and most important class of mock theta functions [Zagier 2009; Zwegers 2002]. Here $x, z \in \mathbb{C}^{*}:=\mathbb{C} \backslash\{0\}$ with neither $z$ nor $x z$ an integral power of $q$, and

$$
j(x, q):=(x)_{\infty}(q / x)_{\infty}(q)_{\infty}
$$

For more on mock theta functions, their remarkable history and modern developments, see [Ono 2009] and [Zagier 2009].

The goal of this paper is to obtain genuine mock theta functions from the Bailey pairs of Bringmann and Kane by first moving a step along the Bailey chain. Applying $(1-3)$ to $\left(a_{n}, b_{n}\right)$ with $(b, c) \rightarrow(-1, \infty)$ and to $\left(\alpha_{n}, \beta_{n}\right)$ with $(b, c) \rightarrow(-q, \infty)$, we obtain the Bailey pairs recorded in the following two lemmas.

Lemma 1.1. The pair $\left(a_{n}^{\prime}, b_{n}^{\prime}\right)$ is a Bailey pair relative to 1 , where

$$
\begin{aligned}
a_{2 n}^{\prime} & =2\left(1-q^{2 n}\right) q^{4 n^{2}-n} \sum_{j=-n}^{n-1} q^{-2 j^{2}-2 j}, \\
a_{2 n+1}^{\prime} & =-2\left(1-q^{2 n+1}\right) q^{4 n^{2}+3 n+1} \sum_{j=-n}^{n} q^{-2 j^{2}}, \\
b_{n}^{\prime} & =\frac{1}{(-q)_{n}} \sum_{j=1}^{n} \frac{(-1)_{j}\left(q ; q^{2}\right)_{j-1}(-1)^{j} q^{\left(\begin{array}{c}
j+1 \\
2
\end{array}\right)}}{(q)_{n-j}(q)_{2 j-1}} .
\end{aligned}
$$

Lemma 1.2. The pair $\left(\alpha_{n}^{\prime}, \beta_{n}^{\prime}\right)$ is a Bailey pair relative to $q$, where

$$
\begin{aligned}
\alpha_{2 n}^{\prime} & =\frac{1}{1-q}\left(q^{4 n^{2}+3 n} \sum_{j=-n}^{n-1} q^{-2 j^{2}-2 j}+q^{4 n^{2}+n} \sum_{j=-n}^{n} q^{-2 j^{2}}\right), \\
\alpha_{2 n+1}^{\prime} & =-\frac{1}{1-q}\left(q^{4 n^{2}+7 n+3} \sum_{j=-n}^{n} q^{-2 j^{2}}+q^{4 n^{2}+5 n+1} \sum_{j=-n-1}^{n} q^{-2 j^{2}-2 j}\right), \\
\beta_{n}^{\prime} & =\frac{1}{(-q)_{n}} \sum_{j=0}^{n} \frac{(-q)_{j}\left(q ; q^{2}\right)_{j}(-1)^{j} q^{\left(\begin{array}{c}
j+1 \\
2
\end{array}\right)}}{(q)_{n-j}(q)_{2 j+1}} .
\end{aligned}
$$


With our main result, we present four mock theta functions arising from the Bailey pairs in Lemmas 1.1 and 1.2. Define

$$
\begin{aligned}
& \theta_{n, p}(x, y, q) \\
& :=\frac{1}{\bar{J}_{0, n p(2 n+p)}} \sum_{r^{*}=0}^{p-1} \sum_{s^{*}=0}^{p-1} q^{n\left(^{r-(n-1) / 2}\right)+(n+p)(r-(n-1) / 2)(s+(n+1) / 2)+n\left(\begin{array}{c}
s+(n+1) / 2 \\
2
\end{array}\right)} \\
& \times \frac{(-x)^{r-(n-1) / 2}(-y)^{s+(n+1) / 2} J_{p^{2}(2 n+p)}^{3} j\left(-q^{n p(s-r)} x^{n} / y^{n}, q^{n p^{2}}\right)}{j\left(q^{p(2 n+p) r+p(n+p) / 2}(-y)^{n+p} /(-x)^{n}, q^{p^{2}(2 n+p)}\right)} \\
& \times \frac{j\left(q^{p(2 n+p)(r+s)+p(n+p)} x^{p} y^{p}, q^{p^{2}(2 n+p)}\right)}{j\left(q^{p(2 n+p) s+p(n+p) / 2}(-x)^{n+p} /(-y)^{n}, q^{p^{2}(2 n+p)}\right)},
\end{aligned}
$$

where $r:=r^{*}+\{(n-1) / 2\}$ and $s:=s^{*}+\{(n-1) / 2\}$ with $0 \leq\{\alpha\}<1$ denoting the fractional part of $\alpha$. Also, $J_{m}:=J_{m, 3 m}$ with $J_{a, m}:=j\left(q^{a}, q^{m}\right)$, and $\bar{J}_{a, m}:=$ $j\left(-q^{a}, q^{m}\right)$.

Theorem 1.3. The following are mock theta functions:

$$
\text { (1-11) } \begin{aligned}
\mathcal{W}_{1}(q): & =\sum_{n \geq j \geq 1} \frac{(-1)_{j}\left(q ; q^{2}\right)_{j-1}(-1)^{j} q^{n^{2}+\left(\begin{array}{c}
j+1 \\
2
\end{array}\right)}}{(-q)_{n}(q)_{n-j}(q)_{2 j-1}} \\
& =4 m\left(-q^{17}, q^{48},-1\right)-4 q^{-5} m\left(-q, q^{48},-1\right)-\frac{2 q^{2} \theta_{3,2}\left(q^{5}, q^{5}, q\right)}{j\left(q, q^{3}\right)},
\end{aligned}
$$

$(1-12) W_{2}(q):=\sum_{n \geq j \geq 1} \frac{\left(q ; q^{2}\right)_{n}(-1)_{j}\left(q ; q^{2}\right)_{j-1}(-1)^{n+j} q^{\left(\begin{array}{c}j+1 \\ 2\end{array}\right)}}{(-q)_{n}(q)_{n-j}(q)_{2 j-1}}$

$$
=4 m\left(-q, q^{8},-1\right)+\frac{2 q \theta_{1,2}\left(-q^{2},-q^{2}, q\right)}{j(-1, q)},
$$

$(1-13) \mathscr{W}_{3}(q):=\sum_{n \geq j \geq 1} \frac{\left(q ; q^{2}\right)_{n}(-1)_{j}\left(q^{2} ; q^{4}\right)_{j-1}(-1)^{n+j} q^{n^{2}+j^{2}+j}}{\left(-q^{2} ; q^{2}\right)_{n}\left(q^{2} ; q^{2}\right)_{n-j}\left(q^{2} ; q^{2}\right)_{2 j-1}}$

$$
=4 m\left(-q, q^{12},-1\right)+\frac{2 q^{3} \theta_{1,1}\left(-q^{7},-q^{7}, q^{4}\right)}{j\left(-q, q^{4}\right)},
$$

(1-14) $\mathscr{W}_{4}(q):=\sum_{n \geq j \geq 0} \frac{(-q)_{j}\left(q ; q^{2}\right)_{j}(-1)^{j} q^{n^{2}+n+\left(\begin{array}{c}j+1 \\ 2\end{array}\right)}}{(-q)_{n}(q)_{n-j}(q)_{2 j+1}}$

$$
=-2 q^{-4} m\left(-q^{5}, q^{48},-1\right)-2 q^{-2} m\left(-q^{11}, q^{48},-1\right)+\frac{\theta_{3,2}\left(q^{3}, q^{3}, q\right)}{j\left(q, q^{3}\right)} \text {. }
$$

It should be noted that the series defining $\mathcal{W}_{2}(q)$ does not converge. However, similar to the sixth order mock theta function $\mu(q)$ [Andrews and Hickerson 1991], 
the sequence of even partial sums and the sequence of odd partial sums both converge. We define $\mathscr{W}_{2}(q)$ as the average of these two values.

To prove Theorem 1.3 we first use the Bailey machinery to express the $\mathscr{W}_{i}$ in terms of Hecke-type double sums $f_{a, b, c}(x, y, q)$, where

$$
f_{a, b, c}(x, y, q):=\sum_{\operatorname{sg}(r)=\operatorname{sg}(s)} \operatorname{sg}(r)(-1)^{r+s} x^{r} y^{s} q^{a\left(\begin{array}{c}
r \\
2
\end{array}\right)+b r s+c\left(\begin{array}{c}
s \\
2
\end{array}\right)} .
$$

Here $x, y \in \mathbb{C}^{*}$ and $\operatorname{sg}(r):=1$ for $r \geq 0$ and $\operatorname{sg}(r):=-1$ for $r<0$. Then we apply recent results of Hickerson and Mortenson [2012] to express the Hecke-type double sums as Appell-Lerch series $m(x, q, z)$ (up to the addition of weakly holomorphic modular forms).

We highlight one connection to classical mock theta functions. Namely, we express the multisum (1-12) in terms of the "eighth order" mock theta functions $S_{1}(q)$ and $T_{1}(q)$, defined by (see [Gordon and McIntosh 2000])

$$
S_{1}(q):=\sum_{n \geq 0} \frac{q^{n(n+2)}\left(-q ; q^{2}\right)_{n}}{\left(-q^{2} ; q^{2}\right)_{n}} \quad \text { and } \quad T_{1}(q):=\sum_{n \geq 0} \frac{q^{n(n+1)}\left(-q^{2} ; q^{2}\right)_{n}}{\left(-q ; q^{2}\right)_{n+1}} .
$$

Corollary 1.4. We have the identity

$$
W_{2}(q)=2 q T_{1}(q)-q S_{1}(q) .
$$

Similar identities involving mock theta functions and multisums were given in [Andrews 2007, Section 13], and more could be deduced from [Bringmann et al. 2010, Theorem 2.4].

The paper proceeds as follows. Some background material on Hecke-type double sums and Appell-Lerch series is collected in Section 2, and Theorem 1.3 and Corollary 1.4 are established in Section 3.

\section{Preliminaries}

We recall some relevant preliminaries. The most important is a result which allows us to convert from the Hecke-type double sums (1-15) to Appell-Lerch series. Define

$$
\begin{aligned}
& g_{a, b, c}\left(x, y, q, z_{1}, z_{0}\right) \\
& :=\sum_{t=0}^{a-1}(-y)^{t} q^{c\left(\begin{array}{c}
t \\
2
\end{array}\right)} j\left(q^{b t} x, q^{a}\right) m\left(-q^{a\left(\begin{array}{c}
b+1 \\
2
\end{array}\right)-c\left(\begin{array}{c}
a+1 \\
2
\end{array}\right)-t\left(b^{2}-a c\right)} \frac{(-y)^{a}}{(-x)^{b}}, q^{a\left(b^{2}-a c\right)}, z_{0}\right) \\
& +\sum_{t=0}^{c-1}(-x)^{t} q^{a\left(\begin{array}{c}
t \\
2
\end{array}\right)} j\left(q^{b t} y, q^{c}\right) m\left(-q^{c\left(\begin{array}{c}
b+1 \\
2
\end{array}\right)-a\left(\begin{array}{c}
c+1 \\
2
\end{array}\right)-t\left(b^{2}-a c\right)} \frac{(-x)^{c}}{(-y)^{b}}, q^{c\left(b^{2}-a c\right)}, z_{1}\right) \text {. }
\end{aligned}
$$


Following Hickerson and Mortenson, we use the term "generic" to mean that the parameters do not cause poles in the Appell-Lerch series or in the quotients of theta functions.

Theorem 2.1 [Hickerson and Mortenson 2012, Theorem 0.3]. Let $n$ and $p$ be positive integers with $(n, p)=1$. For generic $x, y \in \mathbb{C}^{*}$,

$$
f_{n, n+p, n}(x, y, q)=g_{n, n+p, n}(x, y, q,-1,-1)+\theta_{n, p}(x, y, q) .
$$

We shall also require certain facts about $j(x, q), m(x, q, z)$ and $f_{a, b, c}(x, y, q)$. From the definition of $j(x, q)$, we have

$$
j\left(q^{n} x, q\right)=(-1)^{n} q^{-\left(\begin{array}{c}
n \\
2
\end{array}\right) x^{-n} j(x, q)}
$$

where $n \in \mathbb{Z}$ and

$$
j(x, q)=j(q / x, q)=-x j\left(x^{-1}, q\right) .
$$

Next, some relevant properties of the sum $m(x, q, z)$ are given in the following (see (2.2b) of Proposition 2.1 and Theorem 2.3 in [Hickerson and Mortenson 2012]).

Proposition 2.2. For generic $x, z, z_{0} \in \mathbb{C}^{*}$, we have

$$
\begin{gathered}
m(x, q, z)=x^{-1} m\left(x^{-1}, q, z^{-1}\right), \\
m(x, q, z)=m\left(x, q, z_{0}\right)+\frac{z_{0} J_{1}^{3} j\left(z / z_{0}, q\right) j\left(x z z_{0}, q\right)}{j\left(z_{0}, q\right) j(z, q) j\left(x z_{0}, q\right) j(x z, q)} .
\end{gathered}
$$

Finally, two important transformation properties of $f_{a, b, c}(x, y, q)$ are given in the following (see Propositions 5.1 and 5.2 in [Hickerson and Mortenson 2012]).

Proposition 2.3. For $x, y \in \mathbb{C}^{*}$, we have

(2-6) $f_{a, b, c}(x, y, q)$

$$
\begin{aligned}
= & f_{a, b, c}\left(-x^{2} q^{a},-y^{2} q^{c}, q^{4}\right)-x f_{a, b, c}\left(-x^{2} q^{3 a},-y^{2} q^{c+2 b}, q^{4}\right) \\
& -y f_{a, b, c}\left(-x^{2} q^{a+2 b},-y^{2} q^{3 c}, q^{4}\right)+x y q^{b} f_{a, b, c}\left(-x^{2} q^{3 a+2 b},-y^{2} q^{3 c+2 b}, q^{4}\right),
\end{aligned}
$$

$$
f_{a, b, c}(x, y, q)=-\frac{q^{a+b+c}}{x y} f_{a, b, c}\left(q^{2 a+b} / x, q^{2 c+b} / y, q\right) \text {. }
$$

\section{Proof of Theorem 1.3}

Proof of Theorem 1.3. Recall that the goal is to express each double sum $q$-series in terms of Appell-Lerch series. For (1-11), apply Lemma 1.1 and let $b, c \rightarrow \infty$ in 
(1-4) to obtain

$$
\begin{aligned}
\mathscr{W}_{1}(q)= & \sum_{n \geq 0} q^{n^{2}} b_{n}^{\prime}(q)=\frac{1}{(q)_{\infty}} \sum_{n \geq 0} q^{n^{2}} a_{n}^{\prime}(q) \\
= & \frac{1}{(q)_{\infty}}\left(\sum_{n \geq 0} q^{4 n^{2}} a_{2 n}^{\prime}(q)+\sum_{n \geq 0} q^{4 n^{2}+4 n+1} a_{2 n+1}^{\prime}(q)\right) \\
= & \frac{2}{(q)_{\infty}}\left(\sum_{n \geq 0} q^{8 n^{2}-n} \sum_{j=-n}^{n-1} q^{-2 j^{2}-2 j}-\sum_{n \geq 0} q^{8 n^{2}+n} \sum_{j=-n}^{n-1} q^{-2 j^{2}-2 j}\right. \\
& \left.\quad-\sum_{n \geq 0} q^{8 n^{2}+7 n+2} \sum_{j=-n}^{n} q^{-2 j^{2}}-\sum_{n \geq 0} q^{8 n^{2}+9 n+3} \sum_{j=-n}^{n} q^{-2 j^{2}}\right) .
\end{aligned}
$$

After replacing $n$ with $-n$ in the second sum and $n$ with $-n-1$ in the fourth sum, we let $n=(r+s+1) / 2, j=(r-s-1) / 2$ in the first two sums and $n=(r+s) / 2$, $j=(r-s) / 2$ in the latter two sums to find

$$
\begin{aligned}
\mathscr{W}_{1}(q)= & \frac{2 q^{2}}{(q)_{\infty}}\left(\left(\sum_{\substack{r, s \geq 0 \\
r \neq s(\bmod 2)}}-\sum_{\substack{r, s<0 \\
r \neq s(\bmod 2)}}\right) q^{\frac{3}{2} r^{2}+5 r s+\frac{7}{2} r+\frac{3}{2} s^{2}+\frac{7}{2} s}\right. \\
& \left.-\left(\sum_{\substack{r, s \geq 0 \\
r \equiv s(\bmod 2)}}-\sum_{\substack{r, s<0 \\
r \equiv s(\bmod 2)}}\right) q^{\frac{3}{2} r^{2}+5 r s+\frac{7}{2} r+\frac{3}{2} s^{2}+\frac{7}{2} s}\right) \\
= & -\frac{2 q^{2}}{(q)_{\infty}}\left(\left(\sum_{r, s \geq 0}-\sum_{r, s<0}\right) q^{\frac{3}{2} r^{2}+5 r s+\frac{7}{2} r+\frac{3}{2} s^{2}+\frac{7}{2} s}\right) \\
= & -\frac{2 q^{2}}{(q)_{\infty}} f_{3,5,3}\left(q^{5}, q^{5}, q\right) .
\end{aligned}
$$

By Theorem 2.1, (2-1), (2-2) and (2-3), we have

$f_{3,5,3}\left(q^{5}, q^{5}, q\right)$

$=-2 q^{-2} j\left(q, q^{3}\right) m\left(-q^{17}, q^{48},-1\right)+2 q^{-7} j\left(q, q^{3}\right) m\left(-q, q^{48},-1\right)+\theta_{3,2}\left(q^{5}, q^{5}, q\right)$

and so

$$
\mathscr{W}_{1}(q)=4 m\left(-q^{17}, q^{48},-1\right)-4 q^{-5} m\left(-q, q^{48},-1\right)-\frac{2 q^{2} \theta_{3,2}\left(q^{5}, q^{5}, q\right)}{j\left(q, q^{3}\right)} .
$$

For (1-12), apply Lemma 1.1 and let $b=-\sqrt{q}$ and $c=\sqrt{q}$ in (1-4) to get $\mathscr{W}_{2}(q)=\sum_{n \geq 0}(-1)^{n}\left(q ; q^{2}\right)_{n} b_{n}^{\prime}(q)=\frac{\left(q ; q^{2}\right)_{\infty}}{2\left(q^{2} ; q^{2}\right)_{\infty}} \sum_{n \geq 0}(-1)^{n} a_{n}^{\prime}(q)$ 


$$
\begin{aligned}
& =\frac{\left(q ; q^{2}\right)_{\infty}}{2\left(q^{2} ; q^{2}\right)_{\infty}}\left(\sum_{n \geq 0} a_{2 n}^{\prime}(q)-\sum_{n \geq 0} a_{2 n+1}^{\prime}(q)\right) \\
& =\frac{\left(q ; q^{2}\right)_{\infty}}{\left(q^{2} ; q^{2}\right)_{\infty}}\left(\sum_{n \geq 0} q^{4 n^{2}-n} \sum_{j=-n}^{n-1} q^{-2 j^{2}-2 j}-\sum_{n \geq 0} q^{4 n^{2}+n} \sum_{j=-n}^{n-1} q^{-2 j^{2}-2 j}\right. \\
& \left.\quad+\sum_{n \geq 0} q^{4 n^{2}+3 n+1} \sum_{j=-n}^{n} q^{-2 j^{2}}-\sum_{n \geq 0} q^{4 n^{2}+5 n+2} \sum_{j=-n}^{n} q^{-2 j^{2}}\right) .
\end{aligned}
$$

As before, we proceed with

$$
\begin{aligned}
W_{2}(q)= & \frac{\left(q ; q^{2}\right)_{\infty}}{\left(q^{2} ; q^{2}\right)_{\infty}}\left(\left(\sum_{\substack{r \neq s, s \geq 0 \\
r \neq s(\bmod 2)}}-\sum_{\substack{r, s<0 \\
r \neq s(\bmod 2)}}\right) q^{\frac{1}{2} r^{2}+3 r s+\frac{3}{2} r+\frac{1}{2} s^{2}+\frac{3}{2} s+1}\right. \\
& \left.+\left(\sum_{\substack{r, s \geq 0 \\
r \equiv s(\bmod 2)}}-\sum_{\substack{r, s<0 \\
r \equiv s(\bmod 2)}}\right) q^{\left.\frac{1}{2} r^{2}+3 r s+\frac{3}{2} r+\frac{1}{2} s^{2}+\frac{3}{2} s+1\right)}\right) \\
= & \frac{\left(q ; q^{2}\right)_{\infty}}{\left(q^{2} ; q^{2}\right)_{\infty}}\left(\left(\sum_{r, s \geq 0}-\sum_{r, s<0}\right) q^{\left.\frac{1}{2} r^{2}+3 r s+\frac{3}{2} r+\frac{1}{2} s^{2}+\frac{3}{2} s+1\right)}\right) \\
= & \frac{q\left(q ; q^{2}\right)_{\infty}}{\left(q^{2} ; q^{2}\right)_{\infty}} f_{1,3,1}\left(-q^{2},-q^{2}, q\right) .
\end{aligned}
$$

By Theorem 2.1, (2-1) and (2-2), we have

$$
f_{1,3,1}\left(-q^{2},-q^{2}, q\right)=2 q^{-1} j(-1, q) m\left(-q, q^{8},-1\right)+\theta_{1,2}\left(-q^{2},-q^{2}, q\right)
$$

and so

$$
\mathcal{W}_{2}(q)=4 m\left(-q, q^{8},-1\right)+\frac{2 q \theta_{1,2}\left(-q^{2},-q^{2}, q\right)}{j(-1, q)}
$$

For (1-13), apply Lemma 1.1 and let $b=q, c \rightarrow \infty$ and $q \rightarrow q^{2}$ in (1-4) to get

$$
\begin{aligned}
\mathscr{W}_{3}(q) & =\sum_{n \geq 0}(-1)^{n}\left(q ; q^{2}\right)_{n} q^{n^{2}} b_{n}^{\prime}\left(q^{2}\right)=\frac{\left(q ; q^{2}\right)_{\infty}}{\left(q^{2} ; q^{2}\right)_{\infty}} \sum_{n \geq 0}(-1)^{n} q^{n^{2}} a_{n}^{\prime}\left(q^{2}\right) \\
& =\frac{\left(q ; q^{2}\right)_{\infty}}{\left(q^{2} ; q^{2}\right)_{\infty}}\left(\sum_{n \geq 0} q^{4 n^{2}} a_{2 n}^{\prime}\left(q^{2}\right)-\sum_{n \geq 0} q^{4 n^{2}+4 n+1} a_{2 n+1}^{\prime}\left(q^{2}\right)\right)
\end{aligned}
$$




$$
\begin{aligned}
=\frac{2\left(q ; q^{2}\right)_{\infty}}{\left(q^{2} ; q^{2}\right)_{\infty}}\left(\sum_{n \geq 0} q^{12 n^{2}-2 n} \sum_{j=-n}^{n-1} q^{-4 j^{2}-4 j}-\sum_{n \geq 0} q^{12 n^{2}+2 n} \sum_{j=-n}^{n-1} q^{-4 j^{2}-4 j}\right. \\
\left.\quad+\sum_{n \geq 0} q^{12 n^{2}+10 n+3} \sum_{j=-n}^{n} q^{-4 j^{2}}-\sum_{n \geq 0} q^{12 n^{2}+14 n+5} \sum_{j=-n}^{n} q^{-4 j^{2}}\right) .
\end{aligned}
$$

So,

$$
\begin{aligned}
\mathscr{W}_{3}(q)= & \frac{2\left(q ; q^{2}\right)_{\infty}}{\left(q^{2} ; q^{2}\right)_{\infty}}\left(\left(\sum_{\substack{r, s \geq 0 \\
r \neq s(\bmod 2)}}-\sum_{\substack{r, s<0 \\
r \neq s(\bmod 2)}}\right) q^{2 r^{2}+8 r s+5 r+2 s^{2}+5 s+3}\right. \\
& \left.+\left(\sum_{\substack{r, s \geq 0 \\
r \equiv s(\bmod 2)}}-\sum_{\substack{r, s<0 \\
r \equiv s(\bmod 2)}}\right) q^{\left.2 r^{2}+8 r s+5 r+2 s^{2}+5 s+3\right)}\right) \\
= & \frac{2\left(q ; q^{2}\right)_{\infty}}{\left(q^{2} ; q^{2}\right)_{\infty}}\left(\left(\sum_{r, s \geq 0}-\sum_{r, s<0}\right) q^{2 r^{2}+8 r s+5 r+2 s^{2}+5 s+3}\right) \\
= & \frac{2 q^{3}\left(q ; q^{2}\right)_{\infty}}{\left(q^{2} ; q^{2}\right)_{\infty}} f_{1,2,1}\left(-q^{7},-q^{7}, q^{4}\right) .
\end{aligned}
$$

By Theorem 2.1, (2-1), (2-2) and (2-3), we have

$$
f_{1,2,1}\left(-q^{7},-q^{7}, q^{4}\right)=2 q^{-3} j\left(-q, q^{4}\right) m\left(-q, q^{12},-1\right)+\theta_{1,1}\left(-q^{7},-q^{7}, q^{4}\right)
$$

and so

$$
\mathcal{W}_{3}(q)=4 m\left(-q, q^{12},-1\right)+\frac{2 q^{3} \theta_{1,1}\left(-q^{7},-q^{7}, q^{4}\right)}{j\left(-q, q^{4}\right)} .
$$

Finally, for (1-14), apply Lemma 1.2 and let $b, c \rightarrow \infty$ in (1-4) to get

$$
\begin{aligned}
\mathcal{W}_{4}(q)= & \sum_{n \geq 0} q^{n^{2}+n} \beta_{n}^{\prime}(q)=\frac{(1-q)}{(q)_{\infty}} \sum_{n \geq 0} q^{n^{2}+n} \alpha_{n}^{\prime}(q) \\
= & \frac{(1-q)}{(q)_{\infty}}\left(\sum_{n \geq 0} q^{4 n^{2}+2 n} \alpha_{2 n}^{\prime}(q)+\sum_{n \geq 0} q^{4 n^{2}+6 n+2} \alpha_{2 n+1}^{\prime}(q)\right) \\
= & \frac{1}{(q)_{\infty}}\left(\sum_{n \geq 0} q^{8 n^{2}+5 n} \sum_{j=-n}^{n-1} q^{-2 j^{2}-2 j}+\sum_{n \geq 0} q^{8 n^{2}+3 n} \sum_{j=-n}^{n} q^{-2 j^{2}}\right. \\
& \left.\quad-\sum_{n \geq 0} q^{8 n^{2}+13 n+5} \sum_{j=-n}^{n} q^{-2 j^{2}}-\sum_{n \geq 0} q^{8 n^{2}+11 n+3} \sum_{j=-n-1}^{n} q^{-2 j^{2}-2 j}\right) .
\end{aligned}
$$

After replacing $n$ with $-n-1$ in the third and fourth sums, we let $n=(r+s+1) / 2$, $j=(r-s-1) / 2$ in the first and fourth sums and $n=(r+s) / 2, j=(r-s) / 2$ in 
the second and third sums to get

$$
\begin{aligned}
W_{4}(q)= & \frac{1}{(q)_{\infty}}\left(\left(\sum_{\substack{r, s \geq 0 \\
r \neq s(\bmod 2)}}-\sum_{\substack{r, s<0 \\
r \neq s(\bmod 2)}}\right) q^{\frac{3}{2} r^{2}+5 r s+\frac{13}{2} r+\frac{3}{2} s^{2}+\frac{13}{2} s+5}\right. \\
& \left.+\left(\sum_{\substack{r, s \geq 0 \\
r \equiv s(\bmod 2)}}-\sum_{\substack{r, s<0 \\
r \equiv s(\bmod 2)}}\right) q^{\frac{3}{2} r^{2}+5 r s+\frac{3}{2} r+\frac{3}{2} s^{2}+\frac{3}{2} s}\right) \\
= & \frac{1}{(q)_{\infty}}\left(2 q^{13} f_{3,5,3}\left(-q^{25},-q^{29}, q^{4}\right)\right. \\
\quad & \left.\quad+f_{3,5,3}\left(-q^{9},-q^{9}, q^{4}\right)+q^{11} f_{3,5,3}\left(-q^{25},-q^{25}, q^{4}\right)\right) \\
= & \frac{1}{(q)_{\infty}} f_{3,5,3}\left(q^{3}, q^{3}, q\right),
\end{aligned}
$$

where in the last step we have used (2-6) and (2-7). By Theorem 2.1, (2-1), (2-2), (2-3) and (2-4), we have

$$
\begin{aligned}
& f_{3,5,3}\left(q^{3}, q^{3}, q\right) \\
& =-2 q^{-4} j\left(q, q^{3}\right) m\left(-q^{5}, q^{48},-1\right)-2 q^{-2} j\left(q, q^{3}\right) m\left(-q^{11}, q^{48},-1\right)+\theta_{3,2}\left(q^{3}, q^{3}, q\right)
\end{aligned}
$$

and so

$$
\mathscr{W}_{4}(q)=-2 q^{-4} m\left(-q^{5}, q^{48},-1\right)-2 q^{-2} m\left(-q^{11}, q^{48},-1\right)+\frac{\theta_{3,2}\left(q^{3}, q^{3}, q\right)}{j\left(q, q^{3}\right)} .
$$

Proof of Corollary 1.4. Equations (4.36) and (4.38) of [Hickerson and Mortenson 2012] state that

$$
S_{1}(q)=-2 q^{-1} m\left(-q, q^{8},-1\right)+\frac{\bar{J}_{3,8} J_{2,8}^{2}}{q J_{1,8}^{2}}, \quad T_{1}(q)=q^{-1} m\left(-q, q^{8}, q^{6}\right) .
$$

By (1-12), (2-3) and (2-5), the claim is equivalent to the identity

$$
\frac{\bar{J}_{3,8} J_{2,8}^{2}}{J_{1,8}^{2}}+\frac{2 q \theta_{1,2}\left(-q^{2},-q^{2}, q\right)}{j(-1, q)}=\frac{-2 j\left(q^{8}, q^{24}\right)^{3} j\left(-q^{6}, q^{8}\right)}{j\left(q^{6}, q^{8}\right) j\left(-1, q^{8}\right) j\left(-q^{7}, q^{8}\right)} .
$$

We have verified this identity using a MAPLE program [Garvan 2010].

\section{Acknowledgements}

The authors would like to thank Song Heng Chan and Renrong Mao for their helpful comments. 


\section{References}

[Andrews 1986a] G. E. Andrews, "The fifth and seventh order mock theta functions", Trans. Amer. Math. Soc. 293:1 (1986), 113-134. MR 87f:33011 Zbl 0593.10018

[Andrews 1986b] G. E. Andrews, $q$-series: Their development and application in analysis, number theory, combinatorics, physics, and computer algebra, CBMS Regional Conference Series in Mathematics 66, American Mathematical Society, Providence, RI, 1986. MR 88b:11063 Zbl 0594.33001

[Andrews 2001] G. E. Andrews, "Bailey's transform, lemma, chains and tree", pp. 1-22 in Special functions 2000: Current perspective and future directions (Tempe, AZ), edited by J. Bustoz et al., NATO Sci. Ser. II Math. Phys. Chem. 30, Kluwer, Dordrecht, 2001. MR 2004i:33027 Zbl 1005.33005

[Andrews 2007] G. E. Andrews, "Partitions, Durfee symbols, and the Atkin-Garvan moments of ranks", Invent. Math. 169:1 (2007), 37-73. MR 2008d:05013 Zbl 1214.11116

[Andrews and Hickerson 1991] G. E. Andrews and D. Hickerson, "Ramanujan's "lost" notebook, VII: The sixth order mock theta functions", Adv. Math. 89:1 (1991), 60-105. MR 92i:11027 Zbl 0739.11042

[Bringmann and Kane 2011] K. Bringmann and B. Kane, "Multiplicative $q$-hypergeometric series arising from real quadratic fields", Trans. Amer. Math. Soc. 363:4 (2011), 2191-2209. MR 2012f:11208 Zbl 1228.11157

[Bringmann and Ono 2006] K. Bringmann and K. Ono, "The $f(q)$ mock theta function conjecture and partition ranks", Invent. Math. 165:2 (2006), 243-266. MR 2007e:11127 Zbl 1135.11057

[Bringmann and Ono 2010] K. Bringmann and K. Ono, "Dyson's ranks and Maass forms", Ann. of Math. (2) 171:1 (2010), 419-449. MR 2011e:11165 Zbl 05712731

[Bringmann et al. 2010] K. Bringmann, J. Lovejoy, and R. Osburn, "Automorphic properties of generating functions for generalized rank moments and Durfee symbols", Int. Math. Res. Not. 2010:2 (2010), 238-260. MR 2011c:11159 Zbl 1230.05034

[Garvan 2010] F. G. Garvan, "Theta function supplement - preliminary version", online software package, 2010, www.math.ufl.edu/ fgarvan/qmaple/theta-supplement/.

[Gordon and McIntosh 2000] B. Gordon and R. J. McIntosh, "Some eighth order mock theta functions”, J. London Math. Soc. (2) 62:2 (2000), 321-335. MR 2001m:33026 Zbl 1031.11007

[Hickerson and Mortenson 2012] D. Hickerson and E. Mortenson, "Hecke-type double sums, AppellLerch sums, and mock theta functions, I", preprint, 2012. arXiv 1208.1421

[Ono 2009] K. Ono, "Unearthing the visions of a master: Harmonic Maass forms and number theory", pp. 347-454 in Current developments in mathematics (Cambridge, MA, 2008), edited by D. Jerison et al., International Press, Somerville, MA, 2009. MR 2010m:11060 Zbl 1229.11074

[Warnaar 2001] S. O. Warnaar, "50 years of Bailey's lemma”, pp. 333-347 in Algebraic combinatorics and applications (Gößweinstein, 1999), edited by A. Betten et al., Springer, Berlin, 2001. MR 2002g:33020 Zbl 0972.11003

[Zagier 2009] D. Zagier, "Ramanujan's mock theta functions and their applications (after Zwegers and Ono-Bringmann)", pp. 143-164 Astérisque 326, 2009. MR 2011h:11049 Zbl 1198.11046

[Zwegers 2002] S. Zwegers, Mock theta functions, Ph.D. thesis, Universiteit Utrecht, 2002. Zbl 1194.11058 arXiv 0807.4834

Received April 11, 2012. Revised September 19, 2012. 
JEREMY LOVEJOY

CNRS, LIAFA

UNIVERSITÉ DENIS DIDEROT - PARIS 7

CASE 7014

75205 PARIS CEDEX 13

FRANCE

lovejoy@liafa.univ-paris-diderot.fr

ROBERT OSBURN

SCHOOL OF Mathematical SCIENCES

UNIVERSITY COLLEGE DUBLIN

BELFIELD

DUBLIN 4

IRELAND

robert.osburn@ucd.ie 


\title{
PACIFIC JOURNAL OF MATHEMATICS
}

\author{
msp.org/pjm
}

Founded in 1951 by E. F. Beckenbach (1906-1982) and F. Wolf (1904-1989)

\section{EDITORS}

V. S. Varadarajan (Managing Editor)

Department of Mathematics

University of California

Los Angeles, CA 90095-1555

pacific@math.ucla.edu

Paul Balmer

Department of Mathematics

University of California

Los Angeles, CA 90095-1555

balmer@math.ucla.edu

Daryl Cooper

Department of Mathematics

University of California

Santa Barbara, CA 93106-3080 cooper@math.ucsb.edu

Jiang-Hua $\mathrm{Lu}$

Department of Mathematics

The University of Hong Kong

Pokfulam Rd., Hong Kong jhlu@maths.hku.hk
Don Blasius

Department of Mathematics University of California

Los Angeles, CA 90095-1555

blasius@math.ucla.edu

Robert Finn

Department of Mathematics Stanford University

Stanford, CA 94305-2125

finn@math.stanford.edu

Sorin Popa

Department of Mathematics

University of California

Los Angeles, CA 90095-1555

popa@math.ucla.edu

Paul Yang

Department of Mathematics

Princeton University

Princeton NJ 08544-1000

yang@math.princeton.edu

\section{PRODUCTION}

Silvio Levy, Scientific Editor, production@msp.org

\section{SUPPORTING INSTITUTIONS}

ACADEMIA SINICA, TAIPEI

CALIFORNIA INST. OF TECHNOLOGY

INST. DE MATEMÁTICA PURA E APLICADA

KEIO UNIVERSITY

MATH. SCIENCES RESEARCH INSTITUTE

NEW MEXICO STATE UNIV.

OREGON STATE UNIV.

\author{
STANFORD UNIVERSITY \\ UNIV. OF BRITISH COLUMBIA \\ UNIV. OF CALIFORNIA, BERKELEY \\ UNIV. OF CALIFORNIA, DAVIS \\ UNIV. OF CALIFORNIA, LOS ANGELES \\ UNIV. OF CALIFORNIA, RIVERSIDE \\ UNIV. OF CALIFORNIA, SAN DIEGO \\ UNIV. OF CALIF., SANTA BARBARA
}

\author{
Vyjayanthi Chari \\ Department of Mathematics \\ University of California \\ Riverside, CA 92521-0135 \\ chari@math.ucr.edu \\ Kefeng Liu \\ Department of Mathematics \\ University of California \\ Los Angeles, CA 90095-1555 \\ liu@math.ucla.edu \\ Jie Qing \\ Department of Mathematics \\ University of California \\ Santa Cruz, CA 95064 \\ qing@cats.ucsc.edu
}

These supporting institutions contribute to the cost of publication of this Journal, but they are not owners or publishers and have no responsibility for its contents or policies.

See inside back cover or msp.org/pjm for submission instructions.

The subscription price for 2013 is US \$400/year for the electronic version, and \$485/year for print and electronic.

Subscriptions, requests for back issues and changes of subscribers address should be sent to Pacific Journal of Mathematics, P.O. Box 4163, Berkeley, CA 94704-0163, U.S.A. The Pacific Journal of Mathematics is indexed by Mathematical Reviews, Zentralblatt MATH, PASCAL CNRS Index, Referativnyi Zhurnal, Current Mathematical Publications and the Science Citation Index.

The Pacific Journal of Mathematics (ISSN 0030-8730) at the University of California, c/o Department of Mathematics, 798 Evans Hall \#3840, Berkeley, CA 94720-3840, is published monthly except July and August. Periodical rate postage paid at Berkeley, CA 94704, and additional mailing offices. POSTMASTER: send address changes to Pacific Journal of Mathematics, P.O. Box 4163, Berkeley, CA 94704-0163.

PJM peer review and production are managed by EditFLOW ${ }^{\circledR}$ from Mathematical Sciences Publishers.

PUBLISHED BY

mathematical sciences publishers

nonprofit scientific publishing

http://msp.org/

(C) 2013 Mathematical Sciences Publishers 


\section{PACIFIC JOURNAL OF MATHEMATICS}

Volume $264 \quad$ No. $1 \quad$ July 2013

On the center of fusion categories

ALAIN BRUGUIÈRES and ALEXIS VIRELIZIER

Connected quandles associated with pointed abelian groups

W. EdWin Clark, MoHamed ElHamdadi, Xiang-DONG HoU,

MASAHICO SAITO and TIMOTHY YEATMAN

Entropy and lowest eigenvalue on evolving manifolds

HongXin GuO, ROBERT PHILIPOWSKI and ANTON THALMAIER

Poles of certain residual Eisenstein series of classical groups

DIHUA JIANG, BAIYING LIU and LEI ZHANG

Harmonic maps on domains with piecewise Lipschitz continuous metrics 125

HAIGANG LI and CHANGYOU WANG

$q$-hypergeometric double sums as mock theta functions

JEREMY LOVEJOY and ROBERT OSBURN

Monic representations and Gorenstein-projective modules

XIU-HuA LUO and PU ZHANG

Helicoidal flat surfaces in hyperbolic 3-space

Antonio Martínez, JoÃo Paulo dos Santos and Keti

TENENBLAT

On a Galois connection between the subfield lattice and the multiplicative subgroup lattice

JOHN K. MCVEY

Some characterizations of Campanato spaces via commutators on Morrey 221 spaces

Shaoguang Shi and Shanzhen Lu

The Siegel-Weil formula for unitary groups 